\section{Casting nets}

Neutrophils are among the first responders recruited to sites of microbial infection. After activation, neutrophils release proteolytic enzymes and reactive oxygen species that can kill invading microbes. In Science, Brinkman et al. identify extracellular fibers that are rapidly released by neutrophils in response to bacteria or inflammatory mediators. These NETs (neutrophil extracellular traps) comprise chromatin fibers and granule proteins, such as neutrophil elastase and myeloperoxidase. Efficient microbial killing and toxin inactivation occurred within NETs and required chromatin to provide the essential structure for antimicrobial activity. NET formation is independent of apoptosis and, instead, seems to be an innate mechanism for the trapping and exposure of pathogens to high concentrations of toxic antimicrobial products.

$L A D$

Science 303, 1532-1535 (2004)

\section{Pick me}

Data from cellular experiments and computer modeling show that ligation by peptide bound to major histocompatibility complex proteins (pMHCs) marks T cell receptors (TCRs) for internalization and degradation. However, the molecular events that drive this process are unclear. In the Journal of Immunology, La Gruta et al. suggest that an architectural change in the TCR-CD3 complex may be the indicator for eventual TCR downregulation. Ligation of pMHC exposes the TCR-associated CD3 $\zeta \mathrm{N}$ terminus that is normally buried in the complex. This process requires the Src family protein tyrosine kinase and is a late event, presumably to allow signaling events to occur. The change in $\mathrm{CD} 3 \zeta$ and its likely dissociation from the TCR-CD3 complex may further reveal buried lysosomal targeting motifs on other CD3 subunits, which subsequently direct ligated TCR-CD3 complexes for degradation. J. Immunol. 172, 3662-3669 (2004) PTL

\section{The eleventh bell Tolls}

Mammals express ten Toll-like receptors (TLRs) involved in the recognition of pathogen-associated molecular patterns (PAMPs). In Science, Ghosh and colleagues have cloned an additional TLR in mice. TLR11 expression was restricted to macrophages and epithelial cells of the liver, kidney and bladder. This TLR induced NF- $\kappa \mathrm{B}$ activation and a signaling pathway involving MyD88, IRAK and TRAF6. TLR11expressing cells could not respond to known TLR ligands but could respond specifically to uropathogenic bacteria such as Escherichia coli $8 \mathrm{NU}$. Consistently, TLR11-deficient mice were highly susceptible to kidney infection with E. coli 8 NU. Thus, mouse TLR11 probably mediates protection against infection of the urogenital system. The exact ligand remains to be identified and it is unclear so far if humans express TLR11.

Science 303, 1522-1526 (2004)

\section{CHIPS in staph arsenal}

Staphylococcus aureus escapes host defense by producing an unknown factor that inhibits the migration of neutrophils toward chemoattractants such as fMetLeuPhe and the C5a fragment of complement protein C5. In the Journal of Experimental Medicine, de Haas et al. identify and name this factor 'chemotaxis inhibitory protein of

Research notes written by Laurie A. Dempsey, Peter T. Lee and Jamie D.K. Wilson.
S. aureus' (CHIPS). CHIPS specifically bound to neutrophils and monocytes but not lymphocytes. It inhibited fMetLeuPhe- or C5ainduced calcium mobilization. Despite having a greater potency in human than in mouse, CHIPS was able to prevent neutrophil influx in a mouse peritonitis model. The prevalence of the gene encoding CHIPS in clinical isolates of $S$. aureus suggests that it may be a useful target for anti-inflammatory therapy.

J. Exp. Med. 199, 687-695 (2004)

\section{Microbial assassins using TLR4}

Recognition of microbial pathogens by TLR4 usually triggers activation of immune responses that lead to clearance of the infecting pathogen. In Nature, Hsu et al. report that certain virulent strains of bacteria evade immune responses by rerouting TLR4 signaling to induce apoptosis of responding macrophages. Bacillus anthracis, the causal organism of anthrax, as well as several Salmonella and Yersinia species, can induce TLR4 activation of protein kinase $\mathrm{R}$ (PKR), which blocks protein synthesis by inactivating the translation initiation factor elF2 $\alpha$. Bacterial-specific virulence factors blocked the normal TLR4mediated activation of NF- $\mathrm{KB}$ (which induces expression of host survival genes). Macrophages that lacked expression of TLR4, PKR or a mutated elF2 $\alpha$ (S51A) that could not be phosphorylated survived bacterial infections. These results help to explain why these bacteria are such successful pathogens. $L A D$ Nature 428, 341-345 (2004)

\section{MyD88 in cross-priming}

Dendritic cells (DCs) can internalize exogenous antigen and present processed MHC class I epitopes to $\mathrm{CD}^{+}$cytolytic T lymphocytes (CTLs) by the still ill-defined process of cross-presentation. In the Journal of Immunology, Palliser et al. show that the adaptor protein MyD88 is required for DCs to induce CTL effector function in response to exogenous antigen, both in vivo and in vitro. MyD88-deficient DCs were less efficient than wild-type or TLR4-deficient DCs in processing internalized protein for MHC class I presentation. $\mathrm{CD} 8^{+} \mathrm{T}$ cells could proliferate in response to MyD88-deficient DCs that had been given exogenous protein, but had much diminished cytolytic activity and interferon- $\gamma$ production. Thus, the efficiency of processing internalized antigen for CTL cross-priming is sensitive to MyD88 signals.

J. Immunol. 172, 3415-3421 (2004)

\section{Enzyme HINTs for regulation}

MITF is a transcription factor that is essential for mast cell function. HINT, a member of the histidine triad family that can bind nucleotidyl substrates, associates with and suppresses MITF activity. In Immunity, Lee et al. showed that the enzyme lysyl-tRNA (LysRS) is a key regulator of MITF transcriptional activity. LysRS associates with MITF to form a multicomplex that includes HINT. Ap 4 A, a nucleotidyl compound synthesized by LysRS, can bind HINT and induce HINT-MITF dissociation. In immunoglobulin E-activated mast cells, intracellular $\mathrm{Ap}_{4} \mathrm{~A}$ concentrations rapidly increase, therefore reducing the association of HINT with MITF. This renders MITF transcriptionally active. Thus, both LysRS and $\mathrm{Ap}_{4} \mathrm{~A}$ are key regulators of MITF activity in mast cells. $J D K W$ Immunity 20, 145-151 (2004)

$L A D$ 